\title{
Societal Need for Interdisciplinary Ageing Research: An International Alliance of Research Universities "Ageing, Longevity and Health" Stream (IARU-ALH) Position Statement
}

\author{
Nicolas Cherbuin ${ }^{a}$ Katsuya lijima ${ }^{b}$ Sebastiana Kalulac Rahul Malhotrad \\ Lene Juel Rasmussen $^{\mathrm{e}}$ Angelique Chan ${ }^{d, f}$ Louise Lafortune $^{g}$ Sarah Harper $^{\mathrm{h}}$ \\ Xiaoying Zheng ${ }^{i}$ David Lindeman ${ }^{j}$ Erin Walsh $^{a}$ Rafat Hussain ${ }^{a}$ \\ Richard Burns $^{a}$ Maria Kristiansen ${ }^{\mathrm{e}}$ Ikuko Sugawara ${ }^{b}$ Bokyung Son ${ }^{b}$ \\ Tomoki Tanaka $^{b}$ Stefanie Buckner ${ }^{g}$ Jaco Hoffman ${ }^{c, h}$ Marc Combrinck ${ }^{c}$

\begin{abstract}
${ }^{a}$ Centre for Research on Ageing, Health and Wellbeing, Australian National University, Canberra, ACT, Australia; b Institute of Gerontology, Institute for Future Initiatives, The University of Tokyo, Tokyo, Japan; ' Albertina and Walter Sisulu Institute of Ageing in Africa, University of Cape Town, Cape Town, South Africa;

${ }^{\mathrm{d}}$ Duke-National University of Singapore Medical School, Singapore, Singapore; ${ }^{e}$ Center for Healthy Aging, University of Copenhagen, Copenhagen, Denmark; ${ }^{f}$ Department of Sociology, National University of Singapore, Singapore, Singapore; ${ }^{9}$ Cambridge Public Health, University of Cambridge, Cambridge, UK; ${ }^{\text {h}}$ Oxford Institute of Population Health, University of Oxford, Oxford, UK; 'Institute of Population Research, Beijing University, Beijing, China; 'ंCenter for Information Technology Research in the Interest of Society, UC Berkeley, Berkeley, CA, USA
\end{abstract}

\section{Keywords}

Multidisciplinary research $\cdot$ Population ageing $\cdot$ Health . Challenges

\begin{abstract}
Ageing is a global concern with major social, health, and economic implications. While individual countries seek to develop responses to immediate, pressing needs, international attention and collaboration is required to most effectively address the multifaceted challenges and opportunities an ageing global population presents in the longer term. The Ageing, Longevity and Health stream of the International Alliance of Research Universities (IARU-ALH) was built on a solid foundation of first-class interdisciplinary research and on
\end{abstract}

karger@karger.com www.karger.com/bmh

Karger $\stackrel{\text { ' }}{5}$

GOPEN ACCESS
(C) 2021 The Author(s)

Published by S. Karger AG, Basel

This is an Open Access article licensed under the Creative Commons Attribution-NonCommercial-4.0 International License (CC BY-NC) (http://www.karger.com/Services/OpenAccessLicense), applicable to the online version of the article only. Usage and distribution for commercial purposes requires written permission. innovative outreach and communication centres. This interdisciplinary network conducts projects that span biology, medicine, social sciences, epidemiology, public health, poli$c y$, and demography, and actively engages with the public and other societal stakeholders. Here we posit that such international interdisciplinary networks are needed and uniquely placed to address major challenges related to health and ageing and ultimately will produce new understanding and knowledge to promote the awareness of healthy ageing and encourage societal change via novel, science-informed interventions. Global interdisciplinary research presents great potential and opportunities to accelerate our understanding of human ageing and to produce new, more effective solutions to a pressing, complex problem. However, more focused, strategic efforts and invest- 
ments are required in order to deliver on these potentials and reap maximum benefits for individuals and societies. IARU-ALH members are determined to contribute, in collaboration with others, to delivering on this vision.

(c) 2021 The Author(s)

Published by S. Karger AG, Basel

\section{Introduction}

Ageing is a global concern with major social, health, and economic implications. While individual countries seek to develop responses to immediate, pressing needs, international attention and collaboration is required to most effectively address the multifaceted challenges and opportunities an ageing global population presents in the longer term.

The growing proportion of older persons will create pressures on traditional systems of support from families and public services, financial demands on public funds for pensions, and new requirements for healthcare/medical support. Unfortunately, the current COVID-19 pandemic has highlighted how suddenly and unexpectedly these pressures can emerge with older persons being disproportionally at risk of severe chronic disease and of passing away due to COVID-19, and with health and social systems struggling to address their needs.

In order to address these concerns, there is a need not only to better understand ageing at both the individual and societal levels, but also to find more effective ways to mitigate its impact as well as leverage the resources inherent in healthy older populations. In particular, it is widely believed that multiple functional declines, so-called frailty, are inevitable. However, a more recent perspective suggests that there is substantial scope to delay the rate of decline and more effectively mitigate its progression through prevention, technology, healthcare, and social engineering. Simultaneously, it is important to realise and harness the value of older persons in terms of life experiences, wisdom, and their generative potential for younger generations [1].

Internationally, scientific associations including academia and other stakeholders in ageing research have been actively involved in establishing the methods and means to promote interdisciplinary co-operation in the field to achieve a greater, more efficient, and more rapid impact. This requires the development of major crossresearch platforms, which integrate viewpoints from various disciplines spanning the social, medical, biological, and engineering sciences, and the arts and humanities.
As more people live longer, such interdisciplinary research is essential to advance the understanding of the dynamic interplay between the ageing of individuals and their changing technological, social, economic, physical, and global environments. To achieve "Healthy and Active Ageing" [2] and "Successful Ageing" [3] - generally defined as ageing in good health while maintaining independence and staying engaged in social relationships within families and communities across the life course with a high level of well-being - there is a pressing need to develop "comprehensive community-based multi-faceted approaches" and to introduce life course interventions to prevent declines in capacity $[4,5]$.

For these reasons, we as members of the Ageing, Longevity and Health $(\mathrm{ALH})$ stream of the International Alliance of Research Universities (IARU), i.e. IARU-ALH, have come together to develop and concretely implement an international interdisciplinary strategy to address these major societal challenges.

IARU-ALH was built on a solid foundation of firstclass interdisciplinary research and on innovative outreach and communication centres. This interdisciplinary network conducts projects that span biology, medicine, social sciences, epidemiology, public health, policy, and demography, and actively engages with the public and other societal stakeholders. Ultimately, this will contribute to new understanding and knowledge to promote the awareness of healthy ageing and encourage societal change via novel, science-informed interventions. IARUALH brings added value by incorporating basic research, citizen science, international research collaborations, interactions with national and regional stakeholders, communication, education, and entrepreneurship.

We share here a consensus position on the need and likely benefits of such a strategy to promote a broader dialogue, identify barriers and enablers, and chart a way forward to achieve impactful outcomes to improve the quality of life of older persons.

\section{Interdisciplinary Research: A Working Definition}

Interdisciplinary research, as defined by Ferraro and Chan, is "an inquiry involving a plurality of disciplines where disciplinary boundaries are often muted and the joint contributions of the synergy are highlighted" [6]. Thus, by definition, such research, while building upon the expertise of several disciplines, does not highlight the contributions made by a specific discipline (or each involved discipline), rather brings to the fore the implica- 
tions of the collaborative work. The need for and the benefits of interdisciplinary research in general, and specifically in ageing, has been highlighted previously [7-9]. And, over the years, a multitude of ageing research centres or programmes (too many to list here) have identified themselves as being interdisciplinary, bringing together either specialised disciplines such as cell and molecular biology, physiology, and biochemistry within the area of the biology of ageing, or broader scientific disciplines such as sociology, psychology, economics, demography, public health, and epidemiology for studying individual and population ageing.

While this is encouraging, we should like to emphasise three points, which may be overlooked or underrepresented in establishing interdisciplinary research. The first point relates to the need to move beyond specialised interdisciplinary research (e.g. within the basic sciences, or the social sciences) to broader interdisciplinary research. Given the overlap in methodological approaches or tools, scientific language, unit of study, and funding opportunities of various basic science disciplines, and similarly, of social sciences disciplines, and health or medical science disciplines, it is not surprising that interdisciplinary research within each of these "sciences" is more common than that spanning across them. This calls for greater communication among scientists working in varied, seemingly unrelated disciplines, as well as funding opportunities that support broader interdisciplinary research.

The second point is about adding an international perspective to interdisciplinary research. While research methods and findings need to be contextualised to the setting within which they were conducted, especially when studying population or societal ageing, the importance of international collaborative research cannot be undermined. The sharing of knowledge, expertise, and resources across countries and across disciplines can help foster high-quality research in under-resourced settings as well as expand the relevance of the research being conducted. Further, especially in the context of population and societal ageing, international research collaborations can help assess the applicability of underlying theories or mechanisms or hypotheses across countries/cultural settings and the need to modify or adapt them.

The third point is to extend collaboration beyond academic or scientific disciplines. Partnerships with policymakers driving life course ageing policies, communitybased organisations working with and for older persons, and older persons themselves should be strongly considered when designing and conducting interdisciplinary research and assessing its impact. Doing so may allow for better and faster translation of the research findings to the real world - an outcome most of us engaging in interdisciplinary research strive for.

\section{Addressing a Real Need}

The importance of interdisciplinary research and the suboptimal outcomes that can be observed when it is lacking are widespread but perhaps most salient in the domains of population health, and specifically in relation to non-communicable diseases and the ageing process. Since their emergence and throughout the 20th century, the fields of medicine, gerontology, geriatrics, psychiatry, and neurology have mostly considered ageing as a latelife concern, with much of the research focusing on those aged in their 60 s or above. Recent reappraisal has somewhat re-focused attention on the emergence of biological, social, and environmental risk/protective factors for healthy ageing and chronic conditions to mid-life, when signs of disease and senescence start becoming more apparent. Still, the very high likelihood that pathological mechanisms might be first, or at least as much, anchored in childhood or early adulthood, is often not considered.

This prevalent "window-in-time" approach has distracted us away from a holistic understanding of ageing processes and their contributions to chronic conditions, and has probably slowed down the development of population health interventions and policies that can most effectively address the challenges presented by population ageing. This is an area where interdisciplinary research can shine by providing content expertise from one field necessary to resolve important questions that cannot be effectively addressed in another, particularly for disciplines that typically would not interact, such as molecular biology and epidemiology, for example.

The obesity paradox [10] is a good example in the literature of how the lack of interdisciplinarity may lead to misguided interpretation of real effects. Thus, the fact that a higher body mass index in old age is often associated with a lower risk of mortality and morbidity than at mid-life has frequently been interpreted as demonstrating a "protective" effect of adiposity $[11,12]$. While this might seem warranted based purely on statistical associations, it is by far not the only or the most obvious interpretation. This is because fat tissue is known to promote inflammatory and oxidative stress states [13] that are known to damage cells, tissues, and organs, and therefore it would be counterintuitive from an organic chemistry perspective to suggest that excess fat might be protective, 
Table 1. Challenges and barriers to interdisciplinary research

\begin{tabular}{ll}
\hline Challenges & Barriers \\
\hline $\begin{array}{l}\text { Skepticism from non-scientific collaborators is widespread as } \\
\text { they may not have been directly exposed to the scientific process } \\
\text { and may not fully appreciate its relevance to their specialty. }\end{array}$ & $\begin{array}{l}\text { Interdisciplinary research is complex and time-consuming; it } \\
\text { requires time and effort to fully engage another discipline, to } \\
\text { sufficiently understand its language, concepts, substance, and } \\
\text { methods in order to embrace its relevance and application to the } \\
\text { research question. }\end{array}$ \\
\hline
\end{tabular}

Disciplines consider their specialization to be valuable and often perceive themselves as superior to other disciplines.

Criteria for rating the quality of academic departments tend to use a focused approach and are a disincentive to interdisciplinary research collaboration.

There are differences in approaches to knowledge, e.g. the implied There is a lack of specific funding for interdisciplinary research, a superiority of experimental over qualitative approaches. paucity of multidisciplinary training programmes, and a lack of career tracks for junior researchers.

Difficult decisions must be taken when deciding which interdisciplinary centres should participate, which methodologies be implemented, and which collaborations be formed.

Funders are generally unwilling to cover the costs of coordination, which is necessary for truly interdisciplinary research. Such resources are required to support the extended planning period, which characterises this type of research, especially between disciplines with little previous history of intellectual partnerships or where a collaborator may be in a far rural site.

Developing insights from other disciplines and fostering a culture of curiosity are needed for cross-fertilisation.

There is a lack of qualified reviewers of complex interdisciplinary study protocols.

Funding organisations for interdisciplinary research face challenges, which include administrative complexity, time for Academics are trained to become experts in specific subjects and consensus building, and associated risk on the investment. are directed to narrow their focus.

Adapting to and/or combining different publication styles and formats is challenging.

at least as a first explanation [14]. Biologists would also point out that such effects are typically not observed in animal models under highly controlled conditions and probably note that the loss of body mass in ageing is predominantly due to loss of muscle mass not fat mass. This would also be confirmed by gerontologists, who are very familiar with age-related loss of muscle mass and strength (sarcopenia) [15], while epidemiologists might warn against selection bias, reverse causation, and collider stratification [16].

Such nuanced interpretation eventually develops, even within siloed fields, but at high monetary and time costs. Interdisciplinary research can be challenging and disruptive, but it is able to swiftly combine key expertise, generate new concepts, imagine creative solutions that are less likely to emerge from a single field, and thus fast-track translational and transformational research from the bench, to individuals, and to the community.

Interdisciplinary Ageing Research

\section{Challenges/Barriers to Interdisciplinary Research}

In research, a challenge would be defined as a demanding task that calls for special effort or dedication, whereas a barrier is a hurdle that makes it very difficult - but not insurmountable - for groups to work with each other. Ageing is a multidimensional development process, and as argued above, its understanding requires interdisciplinary scholarship [17]. There are, however, several challenges and barriers to interdisciplinary collaboration [17-19] (summarised in Table 1).

\section{Taking International Collaborations to the Next Level with IARU}

As the global focus on ageing increases, IARU-ALH aims to play a central role in international interdisciplinary ageing research, with high-profile initiatives, collabo- 
rations, and networks worldwide. This platform gives access to numerous world-leading research, innovation, and education environments and provides researchers and students with opportunities for mobility, and initiating educational activities to develop innovative services and products for the ageing community.

Since 2006, IARU-ALH has been closely associated with the prestigious IARU (http://www.iaruni.org) initiative, where it is a driving force in the alliance's network. IARU-ALH involvement has led to permanent ageing research-associated activities, such as conferences, seminars, an annual IARU summer school "Interdisciplinary Aspects of Healthy Ageing" (since 2011), an IARU Graduate Student Conference every 2 years (on rotation between IARU members; since 2012), and ongoing research collaborations. These activities have attracted the attention of world research leaders and strengthened relationships among researchers, as well as between researchers and students, in various collaborative projects and courses. In addition to such permanent activities, future activities are expected to include joint research projects and applications targeted at large international funds, as well as faculty exchanges at $\mathrm{PhD}$ and/or postdoc level.

However, some key ingredients are needed to take IARU-ALH to the next level. To translate strong commitments from passionate researchers in the field of ageing and enable concrete large-scale international projects that are attractive to global funding bodies, more foundation structures have to be built. As researchers and leaders, we experience strong pressures to deliver short-term outputs in our local environment. This makes it challenging to divert effort and resources to worthy but more remote objectives for which barriers to delivery are high. Consequently, there is a pressing need for greater investment in seed projects specifically aimed at demonstrating and facilitating international interdisciplinary research across geographical contexts. Such projects produce important pilot data and demonstrate evidence of prior collaboration typically required by major funders, as well as the social glue that supports understanding, shared values, and commitment to common goals.

Additional support is also needed for extended interinstitutional exchanges accessible to academics at all levels, but particularly those early in their career. In spite of the fact that the largest proportion of the world's older population (60+ years old) will reside in Asia and in other developing economies within the next 10 years, the research capacity is far weaker in these regions than in Western countries. There is an urgent need for capacitybuilding among researchers who study ageing-related is- sues to foster evidence-based policy decision-making. IARU representatives are highly respected in their fields and can provide capacity-building opportunities in Asia.

Finally, scientific channels better adapted to the dissemination of interdisciplinary research are needed. The nature of this research means that it cannot conform to norms specific to all its subdisciplines, and therefore dedicated benchmarks, formats, and outlets need to be developed to ensure a high level of knowledge translation.

\section{Conclusion}

Interdisciplinary research presents great potential and opportunities to accelerate our understanding of human ageing and to produce new, more effective solutions to a pressing, complex problem. However, more focused, strategic efforts and investments are required in order to deliver on these potentials and reap maximum benefits for individuals and societies. IARU-ALH members are determined to contribute, in collaboration with others, to delivering on this vision.

\section{Conflict of Interest Statement}

The authors report no conflict of interest.

\section{Funding Sources}

This work is not industry sponsored, and the IARU, which supports this international collaboration, has had no role in any aspect of this article.

\section{Author Contributions}

All authors who contributed significantly to the work have been listed and have approved the final version. N. Cherbuin, K. Iijima, S. Kalula, R. Malhotra, and L.J. Rasmussen made substantial contributions to the conception and design and drafting of the article, and approved the final version. A. Chan, L. Lafortune, S. Harper, X. Zheng, D. Lindeman, E. Walsh, R. Hussain, R. Burns, M. Kristiansen, I. Sugawara, B. Son, T. Tanaka, S. Buckner, J. Hoffman, and M. Combrinck made substantial contributions to the interpretation and critical revision of the article, and approved the final version.
Cherbuin et al. 


\section{References}

1 WHO. Global strategy and action plan on ageing and health. Geneva: WHO; 2017.

2 WHO. Active Ageing: A Policy Framework. Geneva: WHO; 2002

3 Martinson M, Berridge C. Successful aging and its discontents: a systematic review of the social gerontology literature. Gerontologist. 2015 Feb;55(1):58-69.

4 Hornby-Turner YC, Peel NM, Hubbard RE. Health assets in older age: a systematic review. BMJ Open. 2017 May;7(5):e013226.

5 Government Office for Science. Future of an Ageing Population. UK Government; 2016.

6 Ferraro KF. Is Gerontology Interdisciplinary? J Gerontol B Psychol Sci Soc Sci. 2007; 62(1):S2.

7 Pedersen DB. Integrating social sciences and humanities in interdisciplinary research. Palgrave Commun. 2016;2:16036.

8 Kendig H, McDonald P, Piggott J. Introduction A multidisciplinary approach to ageing. In: Kendig HA, McDonald P, Piggott J, editors. Population Ageing and Australia's Future. ANU Press; 2016. pp. 3-18.
9 Keefe JA, Wideman G. Building Capacity for Interdisciplinary Research in Aging. Nova Scotia: Mount Saint Vincent University; 2012.

10 Elagizi A, Kachur S, Lavie CJ, Carbone S, Pandey A, Ortega FB, et al. An Overview and Update on Obesity and the Obesity Paradox in Cardiovascular Diseases. Prog Cardiovasc Dis. 2018 Jul-Aug;61(2):142-50.

11 Auyeung TW, Lee JS, Leung J, Kwok T, Leung PC, Woo J. Survival in older men may benefit from being slightly overweight and centrally obese - a 5-year follow-up study in 4,000 older adults using DXA. J Gerontol A Biol Sci Med Sci. 2010 Jan;65(1):99-104.

12 Hastie CE, Padmanabhan S, Slack R, Pell AC, Oldroyd KG, Flapan AD, et al. Obesity paradox in a cohort of 4880 consecutive patients undergoing percutaneous coronary intervention. Eur Heart J. 2010 Jan;31(2):222-6.
13 Wisse BE. The inflammatory syndrome: the role of adipose tissue cytokines in metabolic disorders linked to obesity. J Am Soc Nephrol. 2004 Nov; 15(11):2792-800.

14 Ellulu MS, Patimah I, Khaza'ai H, Rahmat A, Abed Y. Obesity and inflammation: the linking mechanism and the complications. Arch Med Sci. 2017 Jun;13(4):851-63.

15 Larsson L, Degens H, Li M, Salviati L, Lee YI, Thompson W, et al. Sarcopenia: Aging-Related Loss of Muscle Mass and Function. Physiol Rev. 2019 Jan;99(1):427-511.

16 Banack HR, Stokes A. The 'obesity paradox' may not be a paradox at all. Int J Obes. 2017 Aug;41(8):1162-3.

17 Hennessy $\mathrm{CH}$, Walker A. Promoting multidisciplinary and inter-disciplinary ageing research in the United Kingdom. Ageing Soc. 2011;31(1):52-69.

18 Lynch J. It's not easy being interdisciplinary. Int J Epidemiol. 2006 Oct;35(5):1119-22.

19 Markson E, Stein P. Getting Unstuck: Interdisciplinarity and Aging. Sociol Forum. 2013; 28(4):873-80. 